\title{
Caspase-3 Silencing Attenuates Tumor Repopulation Via Impairing Radiation-Induced DNA Damage Response and Downstream Pathway in Non-Small-Cell Lung Cancer
}

\section{Minghui Zhao}

Shanghai Jiaotong University First People's Hospital

\section{Yiwei Wang}

Shanghai Jiaotong University First People's Hospital

\section{Yucui Zhao}

Shanghai Jiaotong University First People's Hospital

\section{Sijia He}

Shanghai Jiaotong University First People's Hospital

\section{Ruyi Zhao}

Shanghai Jiaotong University First People's Hospital

\section{Yanwei Song}

Shanghai Jiaotong University First People's Hospital

\section{Jin Cheng}

Shanghai Jiaotong University First People's Hospital

\section{Yanping Gong}

Shanghai Jiaotong University First People's Hospital

Jianzhu Xie

Shanghai Jiaotong University First People's Hospital

\section{Yulan Wang}

Shanghai Jiaotong University First People's Hospital

\section{Binjie Hu}

Shanghai Jiaotong University First People's Hospital

\section{Ling Tian}

Shanghai Jiaotong University First People's Hospital

Qian Huang ( $\sim$ huangqian_sjtu@163.com )

Shanghai General Hospital, Shanghai Jiao Tong University School of Medicine

\section{Research}


Keywords: Radiotherapy, Non-small-cell lung cancer, Tumor repopulation, Caspase-3, DNA damage response, Cox-2/PGE2

Posted Date: May 14th, 2020

DOI: https://doi.org/10.21203/rs.3.rs-27514/v1

License: (1) This work is licensed under a Creative Commons Attribution 4.0 International License. Read Full License 


\section{Abstract}

Background: Radiotherapy is an effective treatment on non-small-cell lung cancer (NSCLC). However, radiation-induced dying tumor cells are postulated to generate potent growth signals to stimulate the repopulation of adjacent surviving tumor cells, which may promote tumor recurrence. We investigated the role of caspase-3-centered molecular mechanism in NSCLC repopulation after radiotherapy.

Methods: The stable caspase-3 knockout (Casp3 KO) NSCLC cells were compared with wild-type cells for growth-promoting effect after radiotherapy using in vitro repopulation model. Western blotting, quantitative real-time PCR, enzyme-linked immunosorbent assay and luciferase reporter assay were used to identify the possible molecules and pathway. To elucidate the function of caspase-3 in tumor repopulation, a series of in vitro assays were performed with Casp3 KO NSCLC cells. Finally, tumor cell growth rate of Casp3 $\mathrm{KO}$ and wild-type tumor cells in vivo was tested using xenograft tumor assay and key proteins were further confirmed in tumor tissues with or without radiotherapy.

Results: We found that radiation induced DNA damage response (DDR) and caspase-3 activation, as well as promoted tumor repopulation in NSCLC cells. Unexpectedly, depleting caspase-3 significantly attenuated ataxia-telangiectasia mutated kinase (ATM)/p53-mediated DDR via attenuating endonuclease $G$ (EndoG) nuclear migration, thus decreasing the growth-promoting effect of irradiated dying cells. Moreover, we identified p53 as a regulator of the Cox-2/PGE 2 axis, which was probably involved in caspase-3-centered tumor repopulation after radiotherapy. Additionally, depleting caspase-3 in NSCLC cells showed impaired tumor growth in nude mice model.

Conclusions: Our findings demonstrated that caspase- 3 was implicated in tumor repopulation and that was accompanied by promoting DDR and downstream Cox-2/PGE 2 axis activation in NSCLC cells. This hitherto undescribed signaling pathway mediated by caspase- 3 may deepen insight into the radiobiology and provide therapeutic targets to reduce NSCLC recurrence after radiotherapy.

\section{Background}

Lung cancer ranks the top of cancer related mortality with over a million deaths worldwide annually [1], and non-small-cell lung cancer (NSCLC) accounts for about $85 \%$ of all lung cancers [2]. Radiotherapy is employed as an essential treatment of NSCLC throughout the continuum of care. Despite remarkable advances in treatment in combination with surgical techniques and systemic chemotherapy or radiotherapy, NSCLC still has a dismal prognosis due to therapy resistance and local recurrence, with the median survival less than a year and the 2-year survival rate less than $20 \%$ [3].

Radiotherapy can eradiate the gross of tumor mass, however, it is hard to kill each or every single tumor cell, thus the seeds for tumor relapse are planted. Tumor repopulation can occur attributable to the proliferation of the small number of surviving tumor cells with accelerated pace $[4,5]$. Tumor repopulation is yet an understudied phenomenon, partly due to the negligence of the molecular mechanisms. The possible factors underlying this process include tumor hypoxia [6], inflammation [7], angiogenesis [8] and 
tumor stemness [9]. Therefore, a better understanding of the fundamental process for tumor repopulation after radiotherapy may provide a more efficient therapeutic strategy for NSCLC.

In our previous studies, we showed the paramount importance of apoptosis in tumor repopulation during radiotherapy. The activated caspase-3 not merely implicated in apoptosis, it also promoted the release of growth factors from irradiated dying tumor cells which stimulated growth of adjacent living tumor cells [10]. Among the released growth factors $\mathrm{PGE}_{2}$ was identified as chief culprit since we found that activated caspase 3 cleaved cytosolic calcium-independent phospholipase $\mathrm{A}_{2}\left(\mathrm{iPLA}_{2}\right)$ and then increased the production of arachidonic acid (AA). AA was known to be the precursor of prostaglandin E2 $\left(\mathrm{PGE}_{2}\right)$, whose function was involved in acute inflammatory responses as well as potent mitotic factor [11]. We named this counterintuitive caspase-mediated tumor repopulation mechanism the "Phoenix Rising" pathway. There is increasing recognition that the apoptotic caspase- 3 has a positive role in stimulating cellular proliferation and carcinogenesis. For instance, caspase-3 in dying glioma cells promoted endothelial cell mitosis by activating NF-KB/Cox-2/PGE 2 axis, which established a proangiogenic microenvironment to promote tumor repopulation [12]. Another study demonstrated that activated caspase-3/7 contributed to self-inflicted DNA double-strand breaks (DSBs), resulting in elevated CD133 expression in glioma cancer stem cells (CSCs) [13].

Since radiation kills tumor cells via inducing fatal DNA lesions, we wondered whether the DNA damage repair pathway participates in regulating tumor repopulation. DNA DSBs can arise from exogenous sources such as ionizing radiation or endogenous sources such as reactive oxygen species. Indeed, to handle the DNA lesions, cells in our organism have evolved a complicated network which called DNA damage response (DDR). DDR is composed of numbers of proteins required for cell cycle checkpoints and DNA damage repair. The ataxia-telangiectasia mutated kinase (ATM)/p53 cascade, participated in DNA damage repair, is the most commonly activated pathway in response to DSBs or errors in cell cycle events $[14,15]$. Once DSBs occurs, the sensor kinase ATM is recruited to the damaged sites and autophosphorylated at Ser-1981. Then the activated ATM directly phosphorylates checkpoint kinase 2 (Chk2) on Thr-68 and p53 on Ser-15. Thereafter p53 proteins are stabilized by prevention of ubiquitination and p53-dependent cell cycle arrest, apoptosis or senescence are switched on [15-17]. The irradiated cells use DDR to repair DNA lesions and recover, otherwise, the irreparable damage may trigger cell death process which constitutes the underlying principle of radiotherapy. Moreover, the defects in DDR have been reported to cause genetic instability and drive carcinogenesis [18].

In this study, we designed experiments to examine the unconventional hypothesis in NSCLC that caspase3 coordinates with DDR to be implicated in tumor repopulation during radiotherapy. We presented evidence that ionizing radiation treatment leads to robust activation of DDR and apoptosis. The apoptotic caspase-3 influences DDR and ATM/p53 axis. Unexpectedly, the activated p53 promotes Cox-2/PGE 2 produce in the presence of activated caspase-3 in irradiated NSCLC cells. In the caspase-3 knockout NSCLC cells the Cox-2/PGE 2 produce are remarkably suppressed even p53 is highly expressed. Taken together, ATM/p53/Cox-2/PGE 2 signaling pathway participates in tumor repopulation in NSCLC and this 
effect is strongly interfered by caspase-3. Our findings provide exciting new insights for developing novel therapeutic strategies to counteract recurrence during radiotherapy.

\section{Methods}

\section{Cell culture and irradiation}

Human 293T, non-small-cell lung cancer (NSCLC) cell lines H460 and A549 were purchased from the American Type Culture Collection. 293T cells were cultured in DMEM, H460 and A549 cells were cultured in RPMI 1640 supplemented with $10 \%$ fetal bovine serum (FBS) as well as 100 units/ml penicillin and $100 \mu \mathrm{g} / \mathrm{ml}$ streptomycin (both from Thermo Fisher Scientific) at $37^{\circ} \mathrm{C}$ in humidified incubator with $5 \%$ $\mathrm{CO}_{2}$. Cells or mice were irradiated with a cabinet X-ray generator (Faxitron) operated at $180 \mathrm{kVp}$ and $10 \mathrm{~mA}$ with a dose rate of $3.0 \mathrm{~Gy} / \mathrm{min}$ for the time required to apply a prescribed dose at room temperature.

\section{Lentivirus packaging and transduction}

The pLEX lentiviral system (Open Biosystems) was used to transduce genes into target cells. The firefly luciferase (Fluc) and green fluorescent protein (GFP) fusion gene was kindly provided by Prof. ChuanYuan Li. Fluc and GFP labeled cells (named as Fluc cells) were acquired via lentivirous infection as previous described [10], then cells were cultured in RPMI 1640 supplemented with $10 \%$ FBS and $1 \mu \mathrm{g} / \mathrm{ml}$ puromycin for 2 weeks selection.

\section{Establishment of caspase-3 knockout (Casp3 KO) cells}

Casp3 KO A549 and H460 cells were established by use of CRISPR/Cas9 genome editing system. The Casp3 KO lentivirus-based CRISPR plasmid $[13,19]$ (designated as the Casp3 KO plasmid) was also obtained from Prof. Chuan-Yuan Li. The single-guided RNA (sgRNA) sequence used to disrupt the CASP3 gene is 5'-TAGTTAATAAAGGTATCCA-3'. This plasmid was packaged according to an established protocol [20]. A549 and $\mathrm{H} 460$ cells were seeded into a 6-well plate at a density of $5 \times 10^{5}$, then cells were infected with Casp3 KO plasmid-encoding lentivirus for $24 \mathrm{~h}$ and cultured in RPMI 1640 medium supplemented with $10 \% \mathrm{FBS}, 48 \mathrm{~h}$ after infection, cells were selected in culture medium containing $1 \mu \mathrm{g} / \mathrm{ml}$ puromycin for 2 weeks. Surviving cells were then dissociated by trypsinization (Gibco) to obtain single cells and plated into 96-well plate with 1 cell per well. Single cells derived clones were selected and western blot analysis was used to identify the status of genome editing after an expansion period. Those clones with no detectable caspase-3 signal were chosen for further study.

\section{Cell clonogenic formation assay}


Cells were counted and seeded into 6-well plates. Next, cells were exposed to different radiation doses ( 0 , 2, 4, 6 and 8 Gy with 100 to 10000 cells per well respectively). After 10-14 days, cells were fixed with $4 \%$ paraformaldehyde (Sangon Biotech) and stained with crystal violet (Beyotime). The colonies containing more than 50 cells were scored, experiments were repeated in triplicate. The surviving fraction was calculated as previous described [21].

\section{Flow cytometry}

Cells were treated with 8 Gy radiation for $72 \mathrm{~h}$, then stained with FITC-Annexin V and propidium iodide (PI) using an FITC Annexin V Apoptosis Detection Kit (BD Bioscience) following the manufacturer's instructions. Apoptosis was measured using a BD Accuri C6 flow cytometer.

\section{Western blotting}

Whole cell lysates were prepared in RIPA buffer with protease and phosphatase inhibitors (Roche) at $4^{\circ} \mathrm{C}$. Protein concentrations were determined by using BCA protein assay kit (Thermo Scientific). Western blotting analysis was performed as previously described [22]. Primary antibodies were against $\beta$-tublin, GAPDH, caspase-3, Cleaved caspase-3, Cox-2, ATM, patm-S1981, pchk2-T68, pp53-S15 (\#2128, \#5174, \#9662, \#9664, \#12282, \#2873, \#5883, \#2197, \#9286, Cell Signaling Technology), p53, EndoG, Histone H3 (\#ab1101, \#ab9647, \#ab1791, Abcam) and Chk2 (\#A19543, ABclonal).

\section{Tumor repopulation model and bioluminescence imaging}

In our in vitro repopulation model, a large number $\left(1-2.5 \times 10^{5}\right)$ of irradiated cells (named as feeder cells) were cocultured with a small number (200 or 500) of non-irradiated Fluc cells (named as reporter cells). During coculture period, the culture medium was replaced with fresh 2\% FBS RPMI 1640 every 2 days. After 6-10 days, growth of the Fluc cells was measured by bioluminescence imaging and D-Luciferin potassium $(0.15 \mathrm{mg} / \mathrm{ml}$; Synchem) was added to produce bioluminescence. IVIS Lumina Series III (PerkinElmer) was used to measure bioluminescence imaging.

\section{Quantitative real-time PCR (Q-PCR)}

Total RNA was extracted from cells with RNA-extracting reagent RNAiso Plus and reverse transcribed into cDNA with the PrimeScript ${ }^{\text {TM }}$ RT Master Mix Kit. Q-PCR was performed with TB Green ${ }^{\circledR}$ Premix Ex Taq ${ }^{\text {TM }}$ Kit (\#9109, \#RR036A, \#RR420A, Takara) according to the manufacturer's protocol. The primer of Cox-2 was "GAAGTCCCTGAGCATCTACGG" (forward) and "CCTATCAGTATTAGCCTGCTTGTCT" (reverse). The primer of p53 was "ACCTATGGAAACTACTTCCTGAAA" (forward) and "CTGGCATTCTGGGAGCTTCA" (reverse). The primer of GAPDH was "CCGGGAAACTGTGGCGTGATGG" (forward) and "AGGTGGAGGAGTGGGTGTCGCTGTT" (reverse). GAPDH was used as a loading control. The Q-PCR 
procedure was performed under following conditions: $30 \mathrm{sec}$ at $95^{\circ} \mathrm{C}$, followed by 40 cycles of $5 \mathrm{sec}$ at $95^{\circ} \mathrm{C}$ and $30 \mathrm{sec}$ at $60^{\circ} \mathrm{C}$. The results were obtained at three independent experiments. Relative expression differences were calculated using $2^{-\Delta \Delta C T}$ method.

\section{Transient transfection}

For p53 overexpression, we transiently transfected the pcDNA3-p53 (WT) plasmid in to cells with Lipofectamine 2000 (Life Technologies), following the recommended protocol. The pcDNA3-p53 (WT) plasmid was synthesized by Shanghai HarO Biotech Co., Ltd. and the empty pcDNA3 plasmid (Invitrogen) was used as a control. Cells were incubated with Opti-MEM (Gibco) without FBS during transfections, and transfection medium was replaced with RPMI 1640 after $6 \mathrm{~h}$.

\section{$\mathrm{PGE}_{2}$ enzyme-linked immunosorbent assay (ELISA) assay}

Cells were cultured in RPMI 1640 supplemented with 10\% FBS and treated with 8 Gy radiation. Culture media was removed and replaced with fresh media with $2 \%$ FBS for $16 \mathrm{~h}$ before collection of supernatants at $48 \mathrm{~h}$ after radiation. $\mathrm{PGE}_{2}$ levels in the supernatants were measured by Prostaglandin E2 Express ELISA Kit (Cayman Chemical) according to the manufacturer's protocol.

\section{Reagent}

Celecoxib was obtained from Selleck Chemicals.

\section{Immunofluorescence staining}

Cells were incubated with antibodies against $\mathrm{yH} 2 \mathrm{AX}$, caspase-3 (\#80312, \#9662, Cell Signaling Technology) or EndoG (\#ab9647, Abcam) overnight at $4^{\circ} \mathrm{C}$, then incubated with Alexa Fluor 488 or 594 secondary antibody (Proteintech). Nuclei were counterstained using DAPI reagent (Yeasen). Images were captured using a confocal laser scanning microscope (Leica Microsystems).

\section{Luciferase reporter assay}

The upstream $2 \mathrm{~kb}$ promoter segment of PTGS2 (Cox-2) containing the potential p53 binding site was cloned into GV238 (GeneChem) luciferase reporter vector (PTGS2-WT), and the mutated p53 binding site was also cloned into the same luciferase reporter vector (PTGS2-Mut). P53 overexpression (pcDNA3-p53) plasmid was co-transfected with PTGS2-WT plasmid or PTGS2-Mut plasmid or empty vector in 293T cells for $24 \mathrm{~h}$. Luciferase activity was performed using the dual luciferase reporter assay system (Promega) and normalized to Renilla luciferase activity. 


\section{Xenograft tumor model}

All animal protocols were approved by the Shanghai General Hospital Institutional Animal Care and Use Committee and also in accordance with the guidelines from Directive 2010/63/EU of the European Parliament on the protection of animals used for scientific purposes. Five-week-old BALB/c mice were housed in SPF facilities with free access to normal chow and water. Seven nude mice were injected subcutaneously with wild-type or Casp3 KO H460 cells $\left(5 \times 10^{6}\right.$ cells $)$ into the left or right armpit regions, respectively. Tumor volume (volume $=$ length $\times$ width $^{2} / 2$ ) was determined by calipers every 2 to 3 days. When the mean tumor volumes reached about $2000 \mathrm{~mm}^{3}$, the mice were randomly divided into two groups: 0 Gy radiation $(n=3)$ and 8 Gy radiation $(n=4)$. 48 hours after radiation treatment, all experimental mice were sacrificed and tumor sections were collected for further pathologic examination.

\section{Hematoxylin and eosin (H\&E) and immunohistochemistry (IHC) staining}

$\mathrm{H} \& \mathrm{E}$ and IHC staining were performed as previously described $[23,24]$. The primary antibodies against caspase-3, Cleaved caspase-3, Cox-2, ATM, patm-S1981, pchk2-T68, pp53-S15 (\#9662, \#9664, \#12282, \#2873, \#5883, \#2197, \#9286, Cell Signaling Technology), p53 (\#ab1101, Abcam), Chk2 (\#A19543, ABclonal) were used during the process. Then, the sections were incubated with EnVision ${ }^{\mathrm{TM}} \otimes$ Detection System (GK500705; Gene Tech), counterstained with hematoxylin and visualized using a light microscopy from Leica.

\section{Statistical analysis}

All data were expressed as mean \pm standard error (SE). Statistical analysis was performed using unpaired Student's $t$ test or one-way ANOVA with SPSS 18.0. Statistical significance was determined as $P<0.05$.

\section{Results}

\section{Radiation induces DNA damage, caspase-3 activation and tumor repopulation in NSCLC cells.}

First, we carried out colony formation assay to find the most optimal X-ray dose to produce dying tumor cells. As shown in Fig. S1, the surviving fractions of $8 \mathrm{~Gy}$-irradiated A549 and $\mathrm{H} 460$ cells were $0.043 \% \pm$ $0.014 \%$ and $0.355 \% \pm 0.018 \%$, separately, so we chose $8 \mathrm{~Gy}$ to make dying NSCLC cells. The formation of phosphorylated histone $\mathrm{H} 2 \mathrm{AX}(\mathrm{\gamma H} 2 \mathrm{AX}$ ) foci is a well-recognized marker for DSBs [25], as shown in Fig. $1 \mathrm{~A}$, the levels of $\mathrm{YH} 2 \mathrm{AX}$ foci were greatly increased in the 8 Gy-irradiated cells at $48 \mathrm{~h}$ compared with the control cells. Cell death was measured using Annexin V-FITC and PI double staining by flow cytometry. The percentage of early apoptotic cells (Annexin V-FITC positive and PI negative) and total dead cells (Annexin V-FITC positive) significantly increased in both of the 8 Gy-irradiated A549 and H460 
group on day 3 compared with that in the control group (Fig. 1B-C). Because caspase-3 plays an important role in the execution phase of apoptosis, we next using western blot analysis to clarify whether caspase- 3 was activated following radiation. Profound cleavage of caspase-3 (CC3) was observed in a time-dependent manner in both A549 and H460 cells after 8 Gy radiation (Fig. 1D). We also found the expression of CC3 was markedly enhanced after 8 Gy radiation using immunofluorescence analysis (Fig. 1E). These results demonstrate that $8 \mathrm{~Gy}$ radiation did induce DNA damage accompanied with cell death in NSCLC cells.

To investigate the role of irradiated dying NSCLC cells on living tumor cells, we conducted in vitro repopulation model. Our results initially showed that the luciferase activity of A549 Fluc or H460 Fluc cells was linearly correlated with cell numbers (Fig. S2), thus we could take advantage of luciferase activity to measure Fluc-GFP labeled cells proliferation. Subsequent results demonstrated that $8 \mathrm{~Gy}-$ irradiated A549 feeder cells promoted the proliferation of A549 Fluc reporter cells when compared with A549 Fluc reporter cells growing on sham-irradiated feeder cells and no feeder cells (Fig. 1F). Consistently, 8 Gy-irradiated H460 feeder cells also had potent growth stimulating effect on H460 Fluc reporter cells (Fig. 1F).

\section{Casp3 KO attenuates the growth promoting effect of dying NSCLC cells in vitro.}

Our previous study found that caspase- 3 plays a pivotal role in breast and melanoma tumor cell repopulation $[10,26]$, herein we wondered whether caspase-3 also mediates the growth promoting effect in dying NSCLC cells. By use of CRISPR/Cas9 technology, we generated A549 and H460 cells with genetic ablation of caspase-3 (Casp3 KO). First we performed immunoblotting assays to assess the Casp3 KO efficiency in different mutant single cell clones (data not shown), then selected a clone with sufficient Casp3 KO effect. As shown in Fig. 2A, the levels of caspase-3 were significantly reduced in selected clone compared with the control A549 or $\mathrm{H} 460$ cells (wild-type). We also found the percentage of early apoptotic cells and total dead cells significantly decreased in both of the 8 Gy-irradiated A549/Casp3 KO and $\mathrm{H} 460$ /Casp3 $\mathrm{KO}$ group on day 3 compared with that in the control group (Fig. 2B-C). By using the in vitro repopulation model, we observed that $8 \mathrm{~Gy}$-irradiated Casp3 KO feeder cells significantly diminished the growth-stimulating effect on living reporter cells in both A549 and H460 cells (Fig. 2D).

\section{Activated Cox-2/PGE 2 signaling in dying cells mediates adjacent living tumor cell growth.}

Since Cox-2 plays a pivotal role in the production of the bioactive lipid $\mathrm{PGE}_{2}$, and we previously identified $\mathrm{PGE}_{2}$ as a downstream factor of caspase-3 in tissue regeneration [27], angiogenesis [12] and breast tumor repopulation [10], we therefore conjectured that caspase-3 may also mediate $\mathrm{PGE}_{2}$ production through regulating Cox-2 expression in dying NSCLC cells. The transcription and expression of Cox-2 after 
8 Gy radiation were shown elevated in both A549 and H460 cells in a time-dependent manner by Q-PCR and western blot analysis (Fig. 3B, 3A). However, the transcription and expression of Cox-2 after 8 Gy radiation were markedly restrained due to the Casp3 KO (Fig. 3B, 3A). We then analyzed supernatant $\mathrm{PGE}_{2}$ production from irradiated $\mathrm{A} 549$ and $\mathrm{A} 549$ /Casp3 $\mathrm{KO}$ cells using ELISA assay. As shown in Fig. 3C, the levels of $P G E_{2}$ in 8 Gy-irradiated $A 549$ cells on day 2 increased about fourfold compared to nonirradiated A549 cells. However, secretion of $\mathrm{PGE}_{2}$ was much lower in Casp3 KO cells with or without 8 Gy radiation. Moreover, similar results were found in $\mathrm{H} 460$ and $\mathrm{H} 460 / \mathrm{Casp} 3 \mathrm{KO}$ cells. To determine the importance of $\mathrm{PGE}_{2}$ in regulating the growth-stimulating effect of dying NSCLC cells in vitro, we explored whether the growth of living NSCLC cells was inhibited with downregulation of Cox-2. Treatment with celecoxib $(1 \mu \mathrm{M}$ or $5 \mu \mathrm{M})$, a selective Cox-2 inhibitor, resulted in a dramatic decrease in growth-stimulating effect of dying A549 or H460 feeder cells on A549 Fluc or H460 Fluc reporter cells in a dose-dependent manner (Fig. 3D). In sum, these results demonstrate that $\mathrm{PGE}_{2}$ was involved in caspase-3-mediated NSCLC cells repopulation after radiation.

\section{Casp3 KO attenuates DDR, ATM/p53 signaling and p53- mediated Cox-2 expression in dying NSCLC cells.}

We next tried to elucidate the mechanisms of how caspase-3 enhances Cox-2 expression. The mitochondrial protein endonuclease $\mathrm{G}$ (EndoG) can migrate into nucleus to cleave DNA on development of apoptosis [28]. The distribution of EndoG was determined through immunofluorescence assay. As shown in Fig. 4A, we observed faint staining in the cytoplasmic region of non-irradiated cells, whereas radiotherapy significantly increased nuclear EndoG staining in NSCLC cells. Interestingly, caspase-3 activity seems to be a crucial regulator of EndoG's cytoplasmic to nuclear migration, in irradiated Casp3 KO cells, EndoG's nuclear staining was significantly curbed. Moreover, we found irradiated wild-type cells with nuclear EndoG staining showed higher $\mathrm{YH} 2 \mathrm{AX}$ foci formation. However, the nuclear EndoG and YH2AX foci double staining were mostly absent in irradiated Casp3 KO cells. We also carried out western blot analysis to examine EndoG location before and after radiation in NSCLC cells (Fig. 4B). Consistently, our results showed that radiation-induced EndoG migrated from cytoplasm to nuclear. Moreover, Casp3 $\mathrm{KO}$ in NSCLC cells significantly attenuated the migration.

As ATM is a major sensor of DSBs [29], we assessed protein levels of pATM (S1981) following radiation. We observed a robust ATM phosphorylation in parental A549 cells after radiation. Interestingly, Casp3 KO significantly reduced pATM and total ATM levels after radiation (Fig. 4C). Activated ATM phosphorylates a large chunk of substrates such as Chk2 and p53, thereby propagates the damage signal into numerous cellular pathways [29]. We then checked whether the activated ATM in A549 cells triggered the activation of Chk2 and p53. Indeed, the levels of pChk2 (T68), p53 and pp53 (S15) were much higher after radiation than in the control cells, whereas Casp3 KO significantly reduced the levels of pChk2 (T68), p53 and pp53 (Fig. 4C). Conformably, $\mathrm{H} 460$ cells showed the similar results with or without Casp3 KO after radiation (Fig. 4C). These results suggest that caspase-3 might regulate DDR via ATM/p53 signaling. 
Previously, it has been demonstrated that tumor suppressor p53 could induce Cox-2 expression in response to DNA damage [30]. To elucidate whether p53 regulates the transcription of PTGS2 gene, we first applied the JASPAR database (http://jaspar.genereg.net) to predict p53 binding sites in PTGS2 promoter (Fig. S3). Then we constructed luciferase reporter plasmid encoding the PTGS2 promoter sequence (PTGS2-WT) or mutant sequence (PTGS2-Mut) at region between - $1251 \mathrm{bp}$ and - $1238 \mathrm{bp}$ (Fig. 5A). As shown in Fig. 5B, p53 overexpression cells exhibited higher luciferase activity in PTGS2-WT group compared to controls. However, in PTGS2-Mut group, p53 overexpression did not result in significant difference compared to controls. Next, we examined the transcript level of Cox-2 following overexpression of exogenous p53. As shown in Fig. 5C, $24 \mathrm{~h}$ after transfection in A549 and H460 cells, Cox-2 transcript was induced greater than 10-fold. And we found a severely lower stimulated expression of Cox-2 induced by p53 in Casp3 $\mathrm{KO}$ cell lines (Fig. 5C). We also performed western blotting analysis and results showed that Cox-2 expression was induced in p53 overexpression A549 and H460 cells, but much lower in Casp3 KO cells (Fig. 5D). Based on these results, we elucidated that p53 as a transcription factor activates PTGS2 expression in NSCLC.

\section{Targeting caspase-3 decreases tumorigenicity and radiation induces ATM/p53/Cox-2 axis activation in vivo.}

To determine the effect of Casp3 $\mathrm{KO}$ on tumorigenicity in vivo, we inoculated wild-type and Casp3 KO H460 cells into nude mice subcutaneously. After 22 days, the implanted tumor volumes reached about $2000 \mathrm{~mm}^{3}$ in the wild-type group (the left armpit, 7/7), however, in the Casp3 KO group (the right armpit, $0 / 7)$ no tumor formation was observed (Fig. 6A-C). These data suggested that knockout of caspase-3 restrained tumorigenicity in vivo. Then, mice were randomly divided into 2 groups to be exposed to either 0 Gy or $8 \mathrm{~Gy}$ radiation. Consistent with in vitro results, immunohistochemistry staining showed enhanced levels of Cleaved caspase-3 after radiation for 48 hours (Fig. 6D). Most importantly, increased pATM (S1981)-, pChk2 (T68)-, p53-, pp53 (S15)- and Cox-2-positive cells were observed in the 8 Gy radiation group (Fig. 6D). Moreover, mRNA levels of p53 and Cox-2 were also elevated in tumor tissues after radiation (Fig. S4). Taken together, these experiments supported the notion that activation of caspase-3 along with ATM/p53 signaling pathway are important for Cox-2 induction.

\section{Discussion}

Radiotherapy is employed in cancer treatment to trigger cancer cell death via apoptosis and/or necrosis $[31,32]$. To date, caspase-3 has been considered to be a core executioner caspase cascade responsible for apoptosis [33] and its activity is widely used to evaluate the efficacy of anti-cancer therapeutics [34, 35]. In the present study we demonstrated that caspase-3 coupling DDR in dying NSCLC cells stimulated the proliferation of adjacent living tumor cell, which suggests a favorable role of caspase-3 in tumor relapse following clinical treatment. By comparing Casp3 KO cells with wild-type cells, we provide evidence for the first time that 1 ) apoptotic caspase- 3 could promote DDR by activating the ATM/p53 
signaling pathway; 2 ) activated p53 acted as a positive stimulator in Cox-2/PGE 2 axis which contributes to tumor repopulation of NSCLC.

Given the notion that DNA damage inducers such as radiation usually trigger apoptosis, the fact that apoptotic caspase-3 reversely promoted DSBs following radiation in NSCLC cells is surprising [36]. The most striking aspect of the present study is that our results claimed a novel role of caspase-3, where activated caspase-3 regulates DDR via promoting EndoG nuclear translocation after radiation. Although counterintuitive at first sight, our results are consistent with some studies. Liu et al reported that moderate radiation dose ( $\leq 6 \mathrm{~Gy}$ ) sublethally activated caspase-3, which played a causative role in DNA damage [37]. In Liu's another study, he found that spontaneous cytochrome $C$ leakage ensued caspase- 3 and nucleases activation to cause DNA damage and ATM activation, leading to cancer stemness and tumorigenicity [13]. Regarding to lethally activated caspase-3, earlier research found that in etoposide- or TNFa-treated Hela cells, it could cleave Cdc6 at $D^{290} / S$ and D $442 / G$ sites, driving activation of ATM/ATR kinase and apoptosis [38]. In the field of lethally activated caspase-3 after radiation, we filled the blanks and showed that elevated DDR appeared to depend on caspase-3-mediated ATM/p53 pathway in NSCLC.

As for the downstream effector of caspase-3 mediated tumor repopulation, we paid attention to Cox$2 / \mathrm{PGE}_{2}$ axis, which is involved in tumor initiation, progression and recurrence [39]. In our study caspase-3 deletion impaired Cox-2/PGE 2 expression compared to irradiated wild-type cells, concurrently with weakened tumor repopulation. However, the relationship between p53 and Cox-2 is yet controversial [30, 40-42]. In this study, Cox-2 was induced by exogenous p53 overexpression, as revealed by luciferase reporter assay, suggesting that Cox-2 was a transcriptional target of p53 in NSCLC. Our data showed the induction of Cox-2 was significantly repressed in the absence of caspase-3, indicating a vital role of caspase-3 in Cox-2 regulation, which requires further research. Besides, our results showed that the protein levels of Cleaved caspase-3 was not significantly changed after p53 overexpression in A549 and H460 cells (Fig. 5D). This was consistent with previous studies that restoration of p53 in solid organ tumors mainly caused cell senescence rather than apoptosis $[43,44]$. These results suggested that Cox2/PGE 2 axis works as the downstream target of caspase-3-centered DDR pathway, which participated in radiation-induced tumor repopulation.

Although our study focuses on the Cox-2/PGE 2 as the downstream effector of caspase-3, radiationinduced dying NSCLC cells may also secrete a cocktail of additional growth-stimulating factors, such as VEGF-A, to contribute to tumor relapse after clinical treatment [12, 45]. Also, except for p53/Cox-2 axis, caspase-3 may modulate Cox-2 expression through other pathways. More studies will be needed to examine the additional crucial mechanisms involved in the repopulation of NSCLC after radiotherapy.

\section{Conclusions}

We reported here for the first time that caspase-3 coordinating with DDR exerted a crucial role in tumor repopulation after radiotherapy in NSCLC. Our study revealed a novel mechanism underlying caspase-3- 
centered tumor repopulation, wherein the Cox-2/PGE 2 axis controls the progression of NSCLC after radiotherapy. These molecules may provide promising targets for therapeutic intervention.

\section{Abbreviations}

ATM, ataxia-telangiectasia mutated kinase; Chk2, checkpoint kinase 2; DDR, DNA damage response; DSBs, DNA double-strand breaks; EndoG, endonuclease G; IHC, immunohistochemistry; NSCLC, nonsmall-cell lung cancer; $\mathrm{PGE}_{2}$, Prostaglandin E2

\section{Declarations}

\section{Ethics approval and consent to participate}

All animal experiments (No. 2019A02001) were approved by the Shanghai General Hospital Institutional Animal Care and Use Committee.

\section{Consent for publication}

Not applicable.

\section{Availability of data and materials}

Data and materials are available from the corresponding author on reasonable request.

\section{Competing interests}

The authors declare that they have no competing interests.

\section{Funding}

This study was supported by the National Natural Science Foundation of China (grant 81572951 and 81972843 to Q. Huang).

\section{Authors' contributions}

$\mathrm{QH}$ and $\mathrm{MZ}$ conceived and designed the study; $\mathrm{MZ}, \mathrm{YW}, \mathrm{YZ}$ and $\mathrm{YS}$ performed the experiments; $\mathrm{MZ}, \mathrm{SH}$, $R Z, J C, Y G, J X, Y W$ and $B H$ analyzed the data; $M Z, Q H, Y Z$ and $Y W$ wrote the paper; $L T$ supervised the study. All authors read and approved the final manuscript. 


\section{Acknowledgements}

We thank Wenzhi Tu and Junjian Li (Shanghai General Hospital) for insightful discussions. We also thank the Institute of Translational Medicine at Shanghai General Hospital for providing service and technical support needed for this study.

\section{References}

1. Jemal A, Siegel R, Ward E, Hao Y, Xu J, Thun MJ. Cancer statistics, 2009. CA-Cancer J. Clin. 2009; 59: 225-49.

2. Herbst RS, Heymach JV, Lippman SM. Lung cancer. N. Engl. J. Med. 2008; 359: 1367-80.

3. Dagoglu N, Karaman S, Arifoglu A, Kucucuk S, Oral EN. Definitive radiotherapy in locally advanced non-small cell lung cancer: dose and fractionation. Balk. Med. J. 2014; 31: 278-85.

4. Withers HR, Taylor JM, Maciejewski B. The hazard of accelerated tumor clonogen repopulation during radiotherapy. Acta Oncol. 1988; 27: 131-46.

5. Kim JJ, Tannock IF. Repopulation of cancer cells during therapy: an important cause of treatment failure. Nat. Rev. Cancer. 2005; 5: 516-25.

6. Saggar JK, Tannock IF. Chemotherapy Rescues Hypoxic Tumor Cells and Induces Their Reoxygenation and Repopulation-An Effect That Is Inhibited by the Hypoxia-Activated Prodrug TH302. Clin. Cancer Res. 2015; 21: 2107-14.

7. Reuter S, Gupta SC, Chaturvedi MM, Aggarwal BB. Oxidative stress, inflammation, and cancer: how are they linked? Free Radical Biol. Med. 2010; 49: 1603-16.

8. Asuthkar S, Velpula KK, Nalla AK, Gogineni VR, Gondi CS, Rao JS. Irradiation-induced angiogenesis is associated with an MMP-9-miR-494-syndecan-1 regulatory loop in medulloblastoma cells. Oncogene. 2014; 33: 1922-33.

9. Duru N, Candas D, Jiang G, Li JJ. Breast cancer adaptive resistance: HER2 and cancer stem cell repopulation in a heterogeneous tumor society. J. Cancer Res. Clin. Oncol. 2014; 140: 1-14.

10. Huang Q, Li F, Liu X, Li W, Shi W, Liu FF, et al. Caspase 3-mediated stimulation of tumor cell repopulation during cancer radiotherapy. Nat. Med. 2011;17: 860-6.

11. Wang D, Dubois RN. Prostaglandins and cancer. Gut. 2006; 55: 115-22.

12. Feng $X, Y u Y$, He S, Cheng J, Gong Y, Zhang Z, et al. Dying glioma cells establish a proangiogenic microenvironment through a caspase 3 dependent mechanism. Cancer Lett. 2017; 385: 12-20.

13. Liu X, Li F, Huang Q, Zhang Z, Zhou L, Deng Y, et al. Self-inflicted DNA double-strand breaks sustain tumorigenicity and stemness of cancer cells. Cell Res. 2017; 27: 764-83.

14. Bartek J, Lukas J. Chk1 and Chk2 kinases in checkpoint control and cancer. Cancer Cell. 2003; 3: 421-9.

15. Kastan MB, Bartek J. Cell-cycle checkpoints and cancer. Nature. 2004; 432: 316-23. 
16. Hirao A, Kong YY, Matsuoka S, Wakeham A, Ruland J, Yoshida H, et al. DNA damage-induced activation of p53 by the checkpoint kinase Chk2. Science. 2000; 287: 1824-7.

17. Matsuoka S, Huang M, Elledge SJ. Linkage of ATM to cell cycle regulation by the Chk2 protein kinase. Science. 1998; 282: 1893-7.

18. Ciccia A, Elledge SJ. The DNA damage response: making it safe to play with knives. Mol. Cell. 2010; 40: 179-204.

19. Zhou M, Liu X, Li Z, Huang Q, Li F, Li CY. Caspase-3 regulates the migration, invasion and metastasis of colon cancer cells. Int. J. Cancer. 2018; 143: 921-30.

20. Cong L, Zhang F. Genome engineering using CRISPR-Cas9 system. Methods Mol. Biol. 2015; 1239 : 197-217.

21. Franken NA, Rodermond HM, Stap J, Haveman J, van Bree C. Clonogenic assay of cells in vitro. Nat. Protoc. 2006; 1: 2315-9.

22. Cheng J, Tian L, Ma J, Gong Y, Zhang Z, Chen Z, et al. Dying tumor cells stimulate proliferation of living tumor cells via caspase-dependent protein kinase Cdelta activation in pancreatic ductal adenocarcinoma. Mol. Oncol. 2015; 9: 105-14.

23. Feldman AT, Wolfe D. Tissue processing and hematoxylin and eosin staining. Methods Mol. Biol. 2014; 1180: 31-43.

24. Zhang Z, Wang M, Zhou L, Feng X, Cheng J, Yu Y, et al. Increased HMGB1 and cleaved caspase-3 stimulate the proliferation of tumor cells and are correlated with the poor prognosis in colorectal cancer. J. Exp. Clin. Cancer Res. 2015; 34: 51.

25. Rogakou EP, Pilch DR, Orr AH, Ivanova VS, Bonner WM. DNA double-stranded breaks induce histone H2AX phosphorylation on serine 139. J. Biol. Chem. 1998; 273: 5858-68.

26. Donato AL, Huang Q, Liu X, Li F, Zimmerman MA, Li CY. Caspase 3 promotes surviving melanoma tumor cell growth after cytotoxic therapy. J. Invest. Dermatol. 2014; 134: 1686-92.

27. Li F, Huang Q, Chen J, Peng Y, Roop DR, Bedford JS, et al. Apoptotic cells activate the "phoenix rising" pathway to promote wound healing and tissue regeneration. Sci. Signaling. 2010; 3: ra13.

28. Parrish J, Li L, Klotz K, Ledwich D, Wang X, Xue D. Mitochondrial endonuclease G is important for apoptosis in C. elegans. Nature. 2001; 412: 90-4.

29. Matt S, Hofmann TG. The DNA damage-induced cell death response: a roadmap to kill cancer cells. Cell. Mol. Life Sci. 2016; 73: 2829-50.

30. Han JA, Kim JI, Ongusaha PP, Hwang DH, Ballou LR, Mahale A, et al. P53-mediated induction of Cox2 counteracts p53- or genotoxic stress-induced apoptosis. EMBO J. 2002; 21: 5635-44.

31. Baskar R, Lee KA, Yeo R, Yeoh KW. Cancer and radiation therapy: current advances and future directions. Int. J. Med. Sci. 2012; 9: 193-9.

32. Dewey WC, Ling CC, Meyn RE. Radiation-induced apoptosis: relevance to radiotherapy. Int. J. Radiat. Oncol., Biol., Phys. 1995; 33: 781-96. 
33. Poreba M, Szalek A, Kasperkiewicz P, Rut W, Salvesen GS, Drag M. Small Molecule Active Site Directed Tools for Studying Human Caspases. Chem. Rev. 2015; 115: 12546-629.

34. Dubash SR, Merchant S, Heinzmann K, Mauri F, Lavdas I, Inglese M, et al. Clinical translation of [(18)F]ICMT-11 for measuring chemotherapy-induced caspase 3/7 activation in breast and lung cancer. Eur. J. Nucl. Med. Mol. Imaging. 2018; 45: 2285-99.

35. Cogo F, Poreba M, Rut W, Groborz K, Smyth P, Johnston MC, et al. Development of an advanced nanoformulation for the intracellular delivery of a caspase-3 selective activity-based probe. Nanoscale. 2019; 11: 742-51.

36. Branzei D, Foiani M. Regulation of DNA repair throughout the cell cycle. Nat. Rev. Mol. Cell Biol. 2008; 9: 297-308.

37. Liu X, He Y, Li F, Huang Q, Kato TA, Hall RP, et al. Caspase-3 promotes genetic instability and carcinogenesis. Mol. Cell. 2015; 58: 284-96.

38. Yim H, Hwang IS, Choi JS, Chun KH, Jin YH, Ham YM, et al. Cleavage of Cdc6 by caspase-3 promotes ATM/ATR kinase-mediated apoptosis of HeLa cells. J. Cell Biol. 2006; 174: 77-88.

39. Wu WK, Sung JJ, Lee CW, Yu J, Cho CH. Cyclooxygenase-2 in tumorigenesis of gastrointestinal cancers: an update on the molecular mechanisms. Cancer Lett. 2010; 295: 7-16.

40. Benoit V, de Moraes E, Dar NA, Taranchon E, Bours V, Hautefeuille A, et al. Transcriptional activation of cyclooxygenase-2 by tumor suppressor p53 requires nuclear factor-kappaB. Oncogene. 2006; 25: 5708-18.

41. Subbaramaiah K, Dannenberg AJ. Cyclooxygenase 2: a molecular target for cancer prevention and treatment. Trends Pharmacol. Sci. 2003; 24: 96-102.

42. Gallo O, Schiavone N, Papucci L, Sardi I, Magnelli L, Franchi A, et al. Down-regulation of nitric oxide synthase-2 and cyclooxygenase-2 pathways by p53 in squamous cell carcinoma. Am. J. Pathol. 2003; 163: 723-32.

43. Ventura A, Kirsch DG, McLaughlin ME, Tuveson DA, Grimm J, Lintault L, et al. Restoration of p53 function leads to tumour regression in vivo. Nature. 2007; 445: 661-5.

44. Xue W, Zender L, Miething C, Dickins RA, Hernando E, Krizhanovsky V, et al. Senescence and tumour clearance is triggered by p53 restoration in murine liver carcinomas. Nature. 2007; 445: 656-60.

45. Cheng J, He S, Wang M, Zhou L, Zhang Z, Feng X, et al. The Caspase-3/PKCdelta/Akt/VEGF-A Signaling Pathway Mediates Tumor Repopulation during Radiotherapy. Clin. Cancer Res. 2019; 25: 3732-43.

\section{Figures}


A
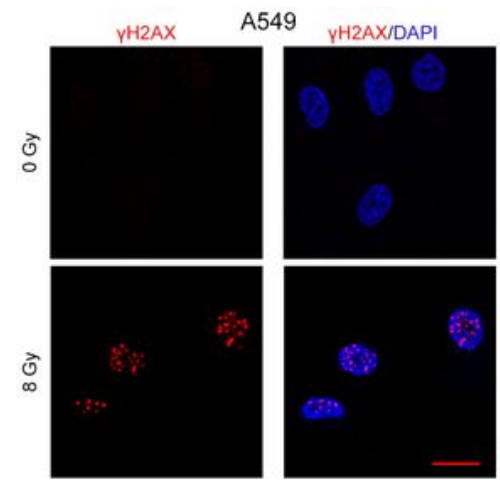

B

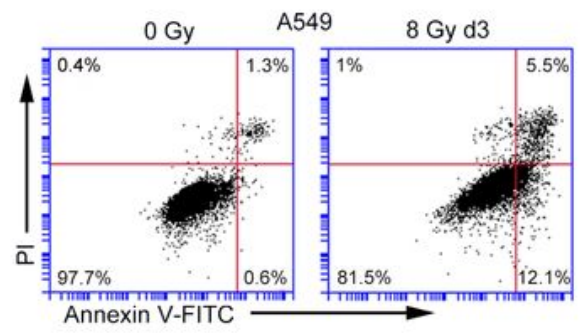

C

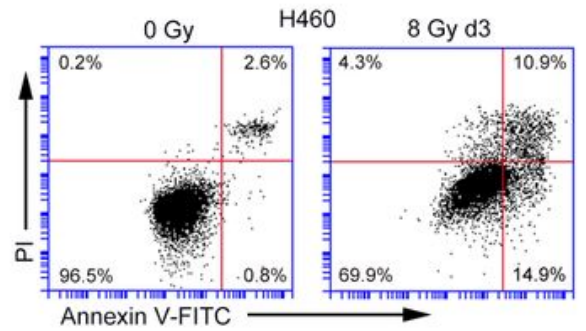

D

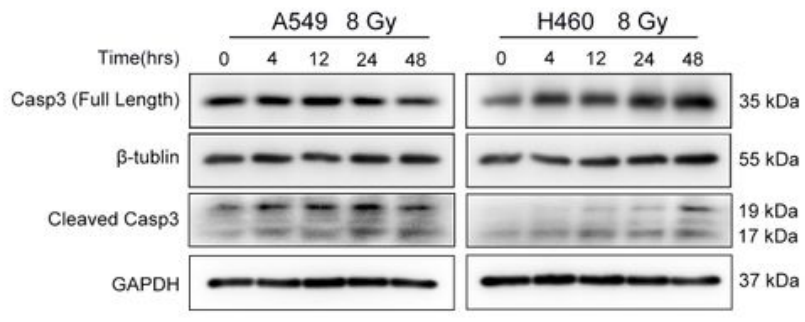

F

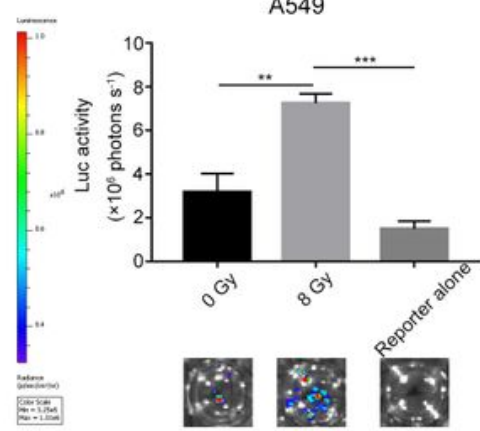

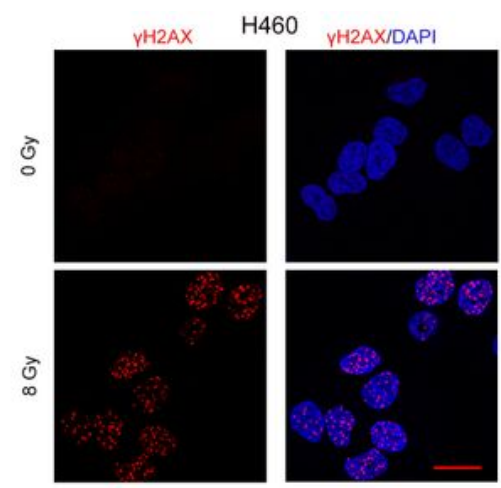

A549
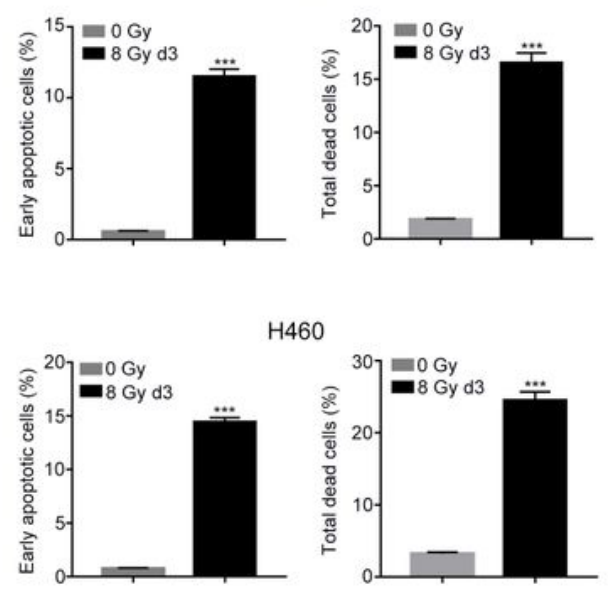

E
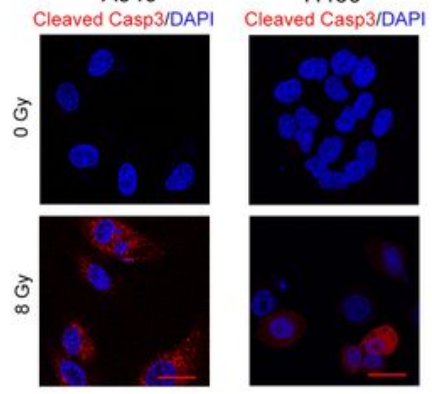

H460

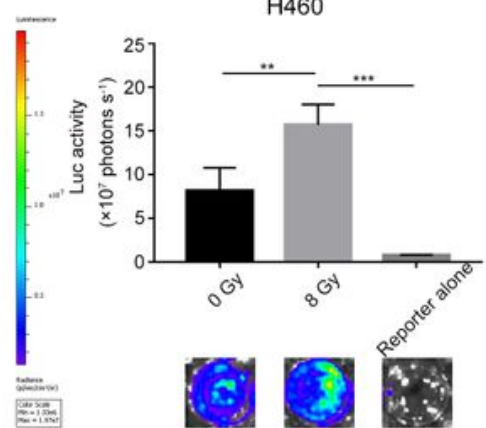

\section{Figure 1}

Radiation induces DNA damage, caspase-3 activation and tumor repopulation in NSCLC cells. A. Confocal immuno-staining images of $\mathrm{YH} 2 \mathrm{AX}$ foci in A549 and $\mathrm{H} 460$ cells following $8 \mathrm{~Gy}$ radiation at 48 hours. Scale bars: $25 \mu \mathrm{m}$. B-C. Left panel, flow cytometry analysis of A549 (B) or H460 (C) cell death after 0 Gy or 8 Gy radiation at day 3. Apoptosis was monitored by Annexin V/PI double staining. Right panel, quantitative analysis of early apoptosis and total cell death in 0 Gy- or 8 Gy-irradiated A549 (B) or H460 
(C) cells ( $* \star \star p<0.001$, Student's $t$ test, $n=3$ ). D. Cleaved caspase 3 induced by 8 Gy radiation was determined by western blotting, $\beta$-tublin and GAPDH served as loading control. E. Representative confocal images for immunofluorescence staining of cleaved caspase-3 in A549 and H460 cells following 8Gy radiation at day 3 . Scale bars: $25 \mu \mathrm{m}$. F. 8 Gy-irradiated NSCLC cells promoted the growth of living NSCLC reporter cells. Upper panel, luciferase activities showed the growth of A549 Fluc or H460 Fluc cells which were seeded with 0 Gy or 8 Gy radiation treated NSCLC cells or alone. Lower panel, representative bioluminescence images ( ${ }^{* *} p<0.01,{ }^{* *} p<0.001$, one-way ANOVA, $\left.n=4\right)$.

A

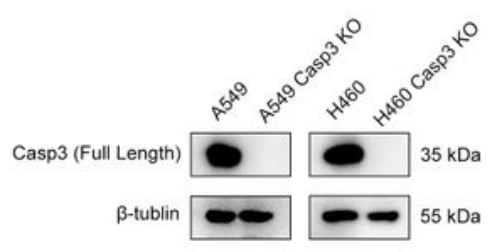

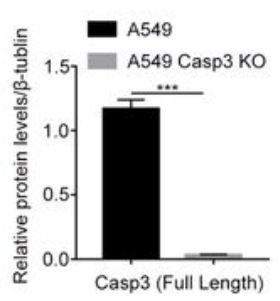

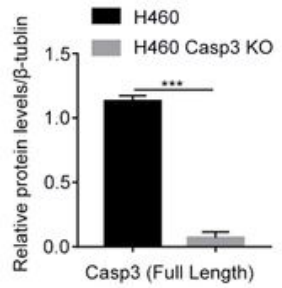

B

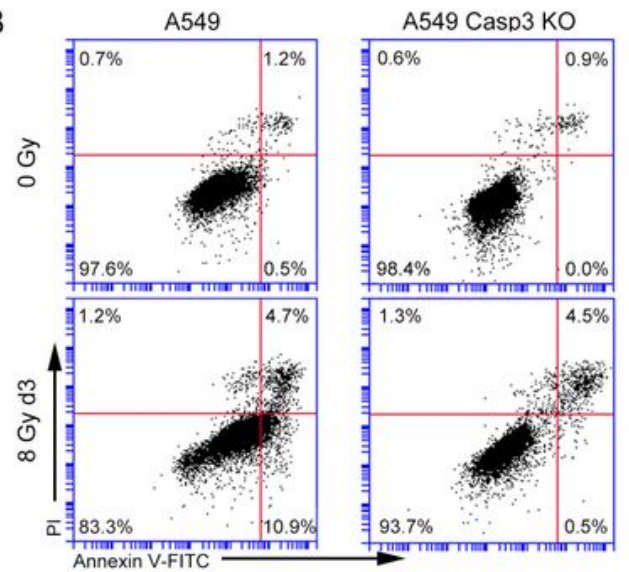

C

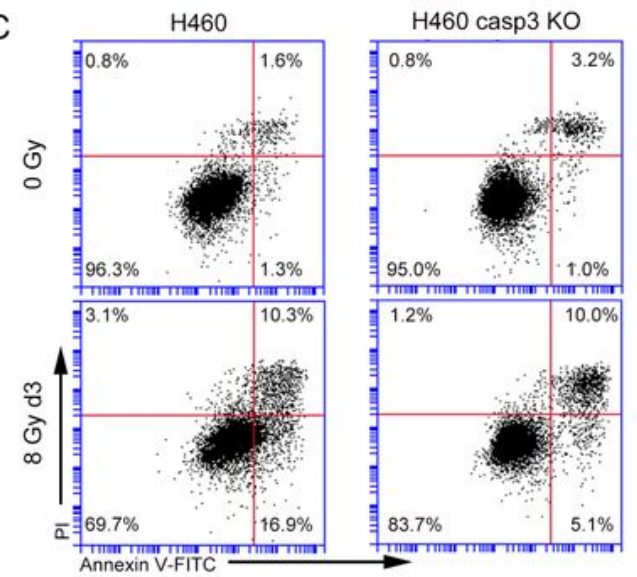

D

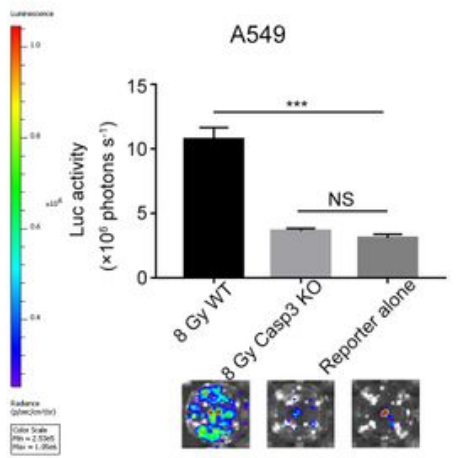

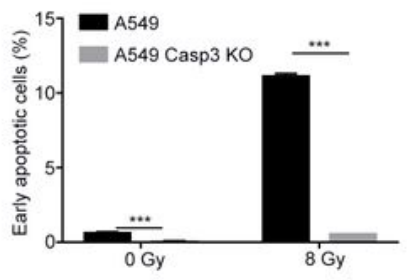
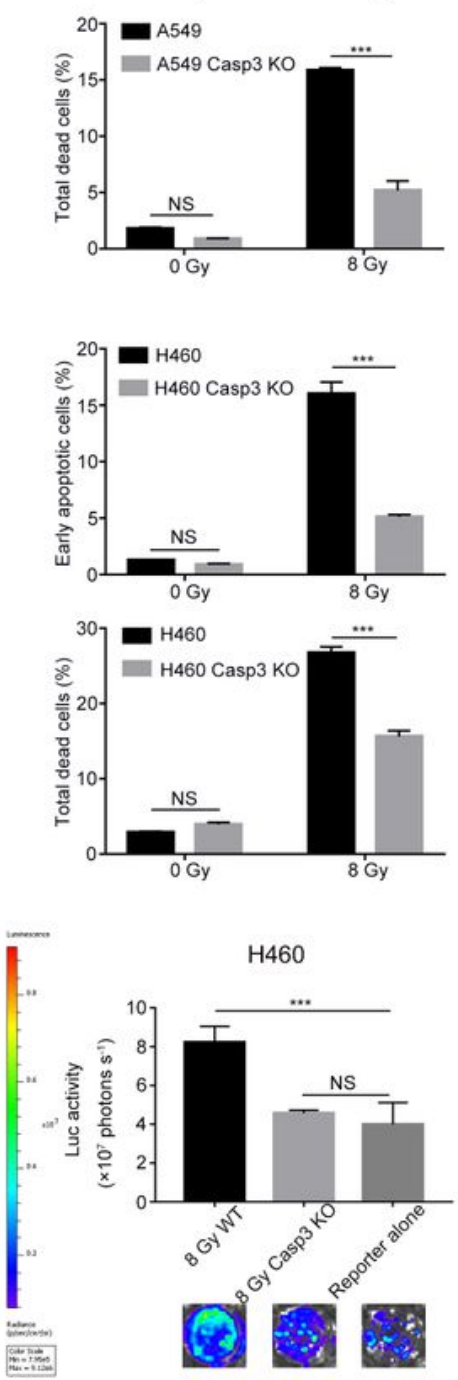


\section{Figure 2}

Casp3 KO attenuates radiation-induced apoptosis and the growth promoting effect of dying NSCLC cells. A. Western blotting analysis of the expression of caspase-3 in Casp3 KO A549 and H460 cells via CRISPR/Cas9 system. $\beta$-tublin was used as a loading control ( $* \star \star p<0.001$, Student's $t$ test, $n=3)$. B-C. Left panel, flow cytometry analysis of cell death manner in A549 and A549/Casp3 KO (B) or H460 and H460/Casp3 KO (C) following radiation. Cell apoptotic death events were determined by Annexin V-/PI double staining. Right panel, quantitative analysis of early apoptosis and total cell death in $0 \mathrm{~Gy}$ - or 8 Gyirradiated control and A549/Casp3 KO (B) or H460/Casp3 KO (C) cells ( $* \star \star p<0.001$, NS = not significant, Student's $t$ test, $n=3)$. D. Casp3 KO significantly decreased the growth promoting effect of 8 Gy-irradiated NSCLC cells on living NSCLC reporter cells. Upper panel, luciferase activities showed the growth of A549 Fluc or H460 Fluc cells which were seeded with 8 Gy-irradiated wild-type or Casp3 KO cells or alone. Lower panel, representative bioluminescence images ( $* \star \star p<0.001$, NS = not significant, one-way ANOVA, $n$ $=4)$. 

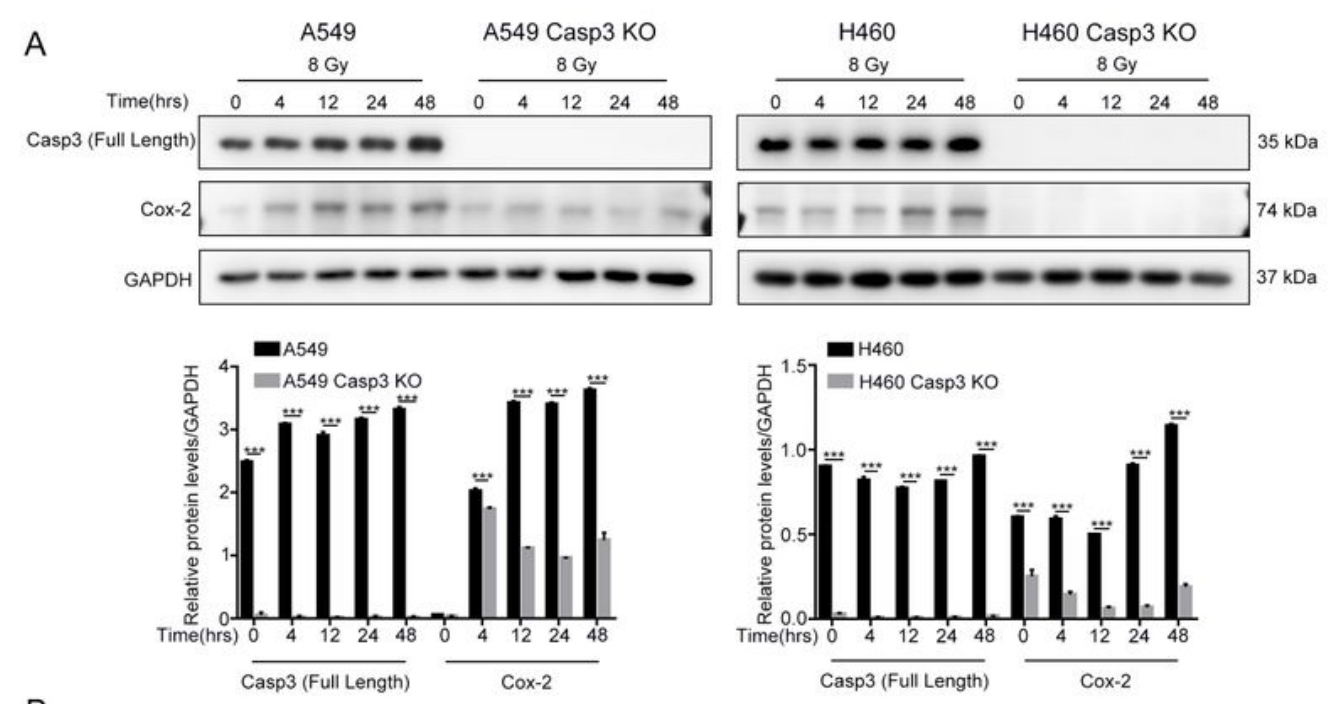

B
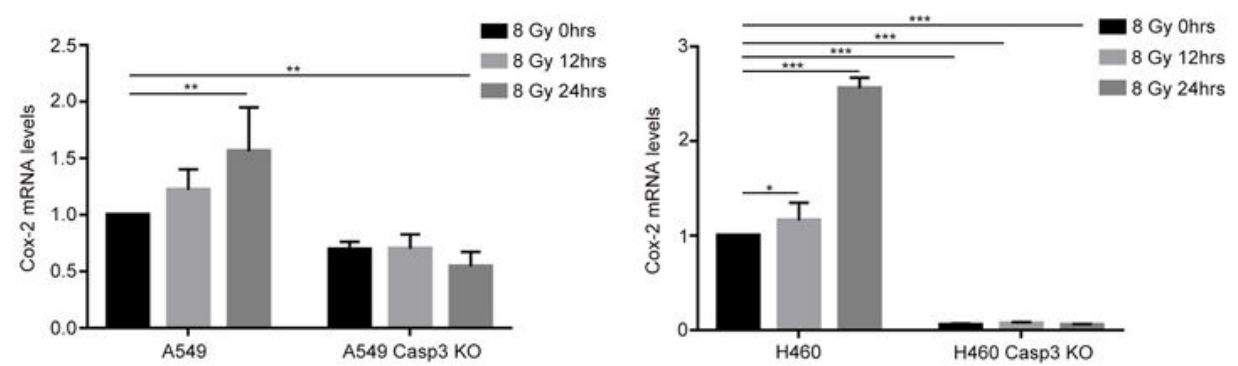

C
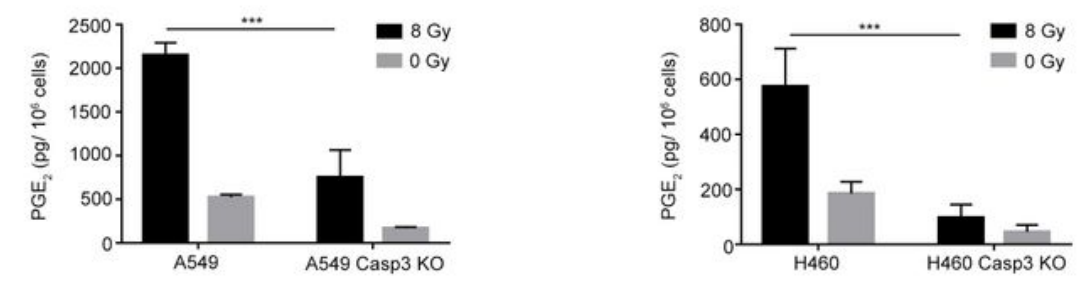

D
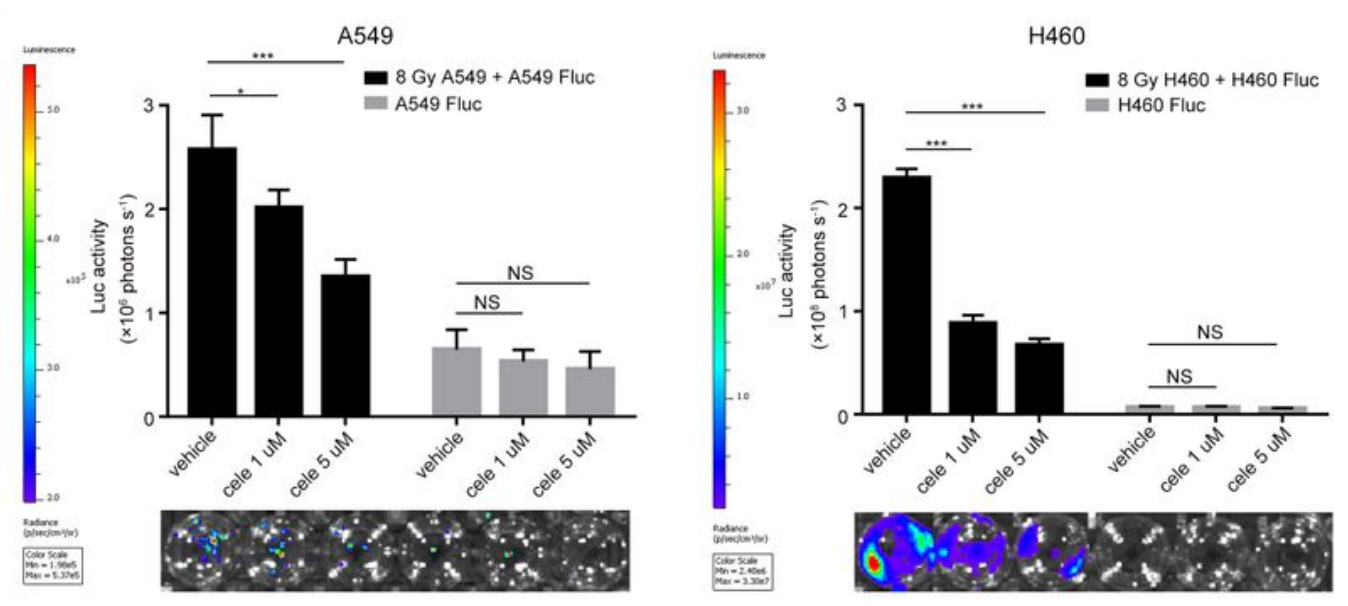

\section{Figure 3}

Caspase-3-dependent PGE2 production in dying NSCLC cells induces tumor repopulation. A. Western blot analysis of Cox-2 levels at various time after 8 Gy radiation in wild-type and Casp3 KO NSCLC cells $(* \star * p<0.001$, one-way ANOVA, $n=3)$. B. Quantitative PCR (qPCR) analysis of Cox-2 in wild-type and Casp3 KO NSCLC cells at indicated time after 8 Gy radiation $\left({ }^{*} p<0.05,{ }^{*} p<0.01,{ }^{* \star *} p<0.001\right.$, one-way ANOVA, $n$ = 3). C. The levels of PGE2 in culture supernatants of wild-type and Casp3 KO NSCLC cells at 48 hours 
after 8 Gy radiation were measured by ELISA ( ${ }^{* \star} p<0.001$, one-way ANOVA, $\left.n=3\right)$. D. A selective Cox-2 inhibitor, celecoxib, abrogated the pro-proliferation effects of dying NSCLC cells on Fluc cells in a dosedependent manner $\left({ }^{*} p<0.05,{ }^{* *} p<0.001, \mathrm{NS}=\right.$ not significant, one-way ANOVA, $\left.\mathrm{n}=4\right)$.
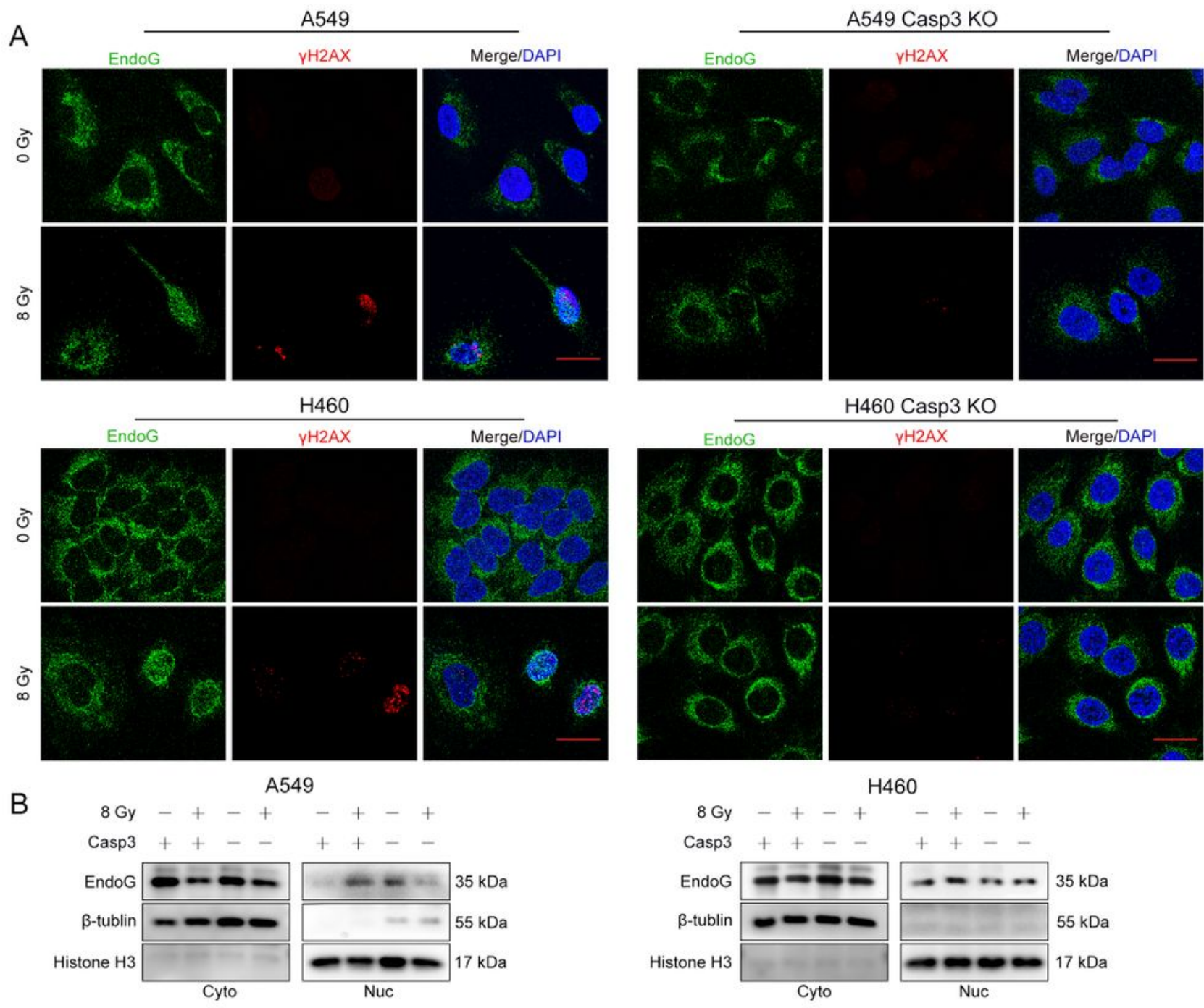

A549

$\mathrm{H} 460$
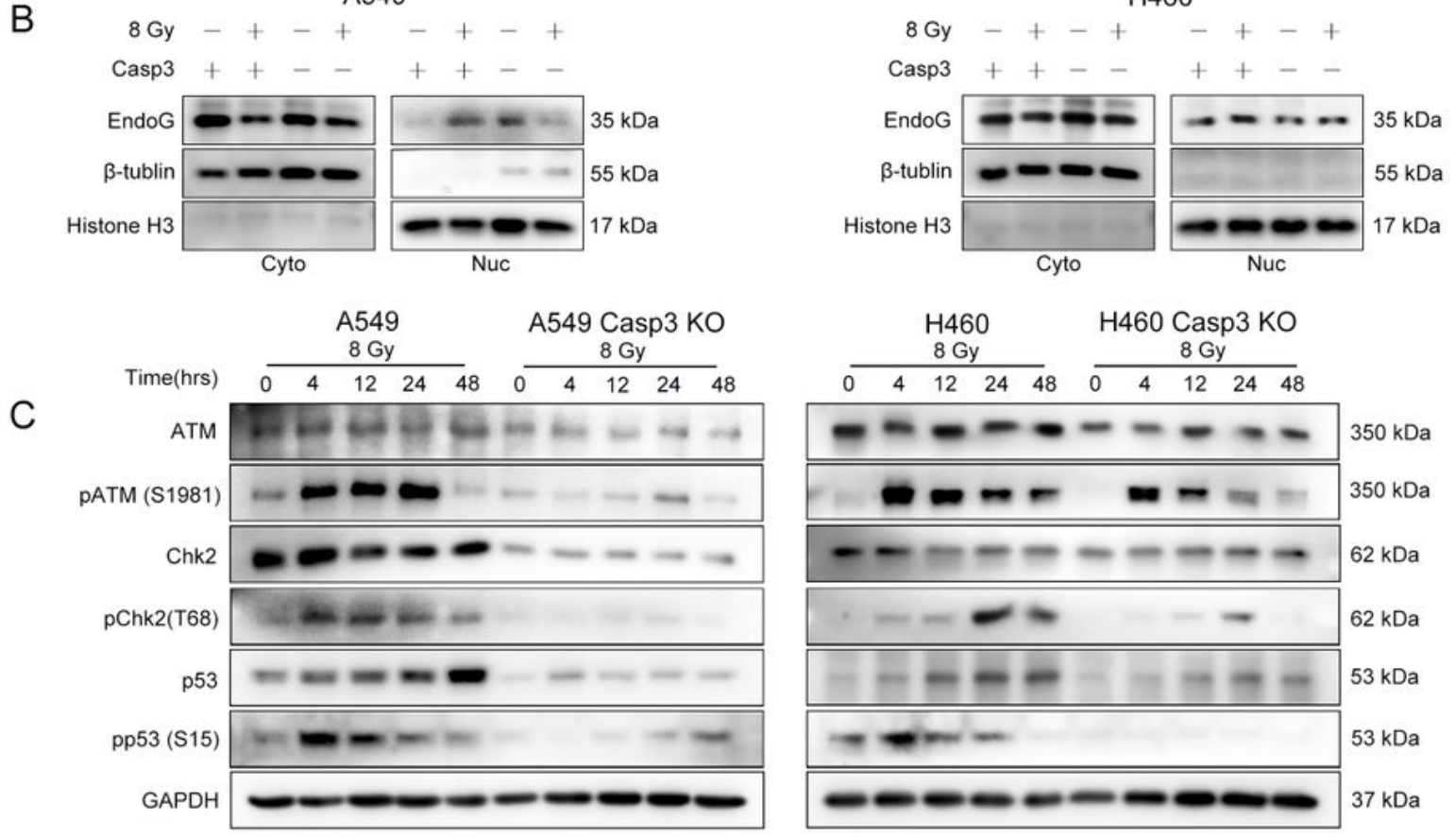

Figure 4

Casp3 KO attenuates DDR via ATM/p53 signal in irradiated NSCLC cells. A. Immunofluorescence costaining of EndoG and yH2AX foci in 8Gy-irradiated wild-type and Casp3 KO NSCLC cells at 48 hours. 
Scale bars: $25 \mu \mathrm{m}$. B. Western blot analysis of EndoG in the cytoplasmic and nuclear fractions of 8Gyirradiated wild-type and Casp3 KO NSCLC cells at 48 hours. $\beta$-tublin and Histone H3 were used as cytoplasmic and nuclear loading controls, respectively. C. The expression levels of DDR-related proteins ATM, pATM (S1981), Chk2, pChk2 (T68), p53, pp53 (S15) were measured by western blot at indicated time after 8 Gy radiation in wild-type and Casp3 KO NSCLC cells. GAPDH was used as a loading control.

A

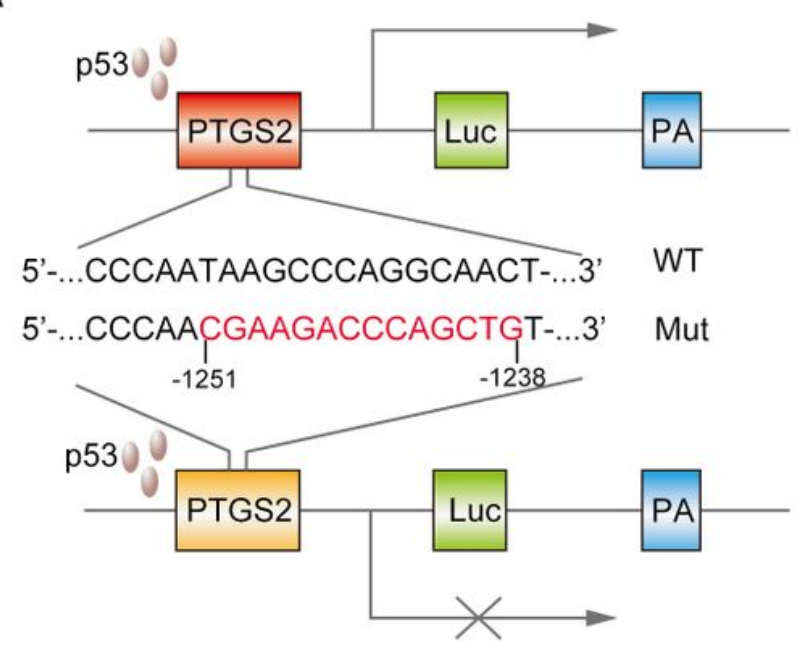

C

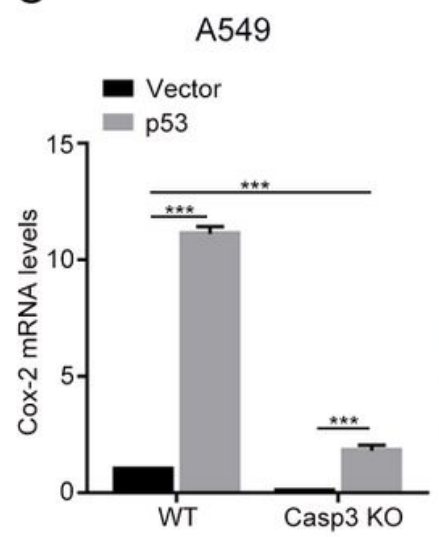

B

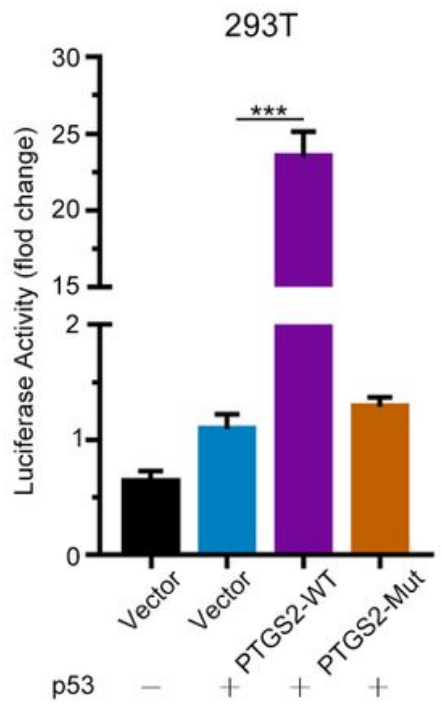

\section{Figure 5}

P53 contributes to Cox-2 induction in NSCLC cells. A. A schematic representation of luciferase reporter plasmid with the PTGS2 promoter sequence (PTGS2-WT) or mutant sequence (PTGS2-Mut). B. P53dependent induction of PTGS2 promoter activity was revealed by luciferase assay. P53 overexpression plasmid was co-transfected with PTGS2-WT plasmid or PTGS2-Mut plasmid or vector alone into 293T, and pGMR-TK reporter as internal transfection standard ( ${ }^{* \star} p<0.001$, one-way ANOVA, $n=3$ ). C-D. QPCR and western blot showed the mRNA and protein levels of Cox-2 were elevated by induction of exogenous p53 in wild-type and Casp3 KO NSCLC cells. Total RNA and proteins were extracted after transfection for 24 hours and 48 hours, respectively ( ${ }^{\star * \star} p<0.001$, Student's $t$ test, $n=3$ ). 
A
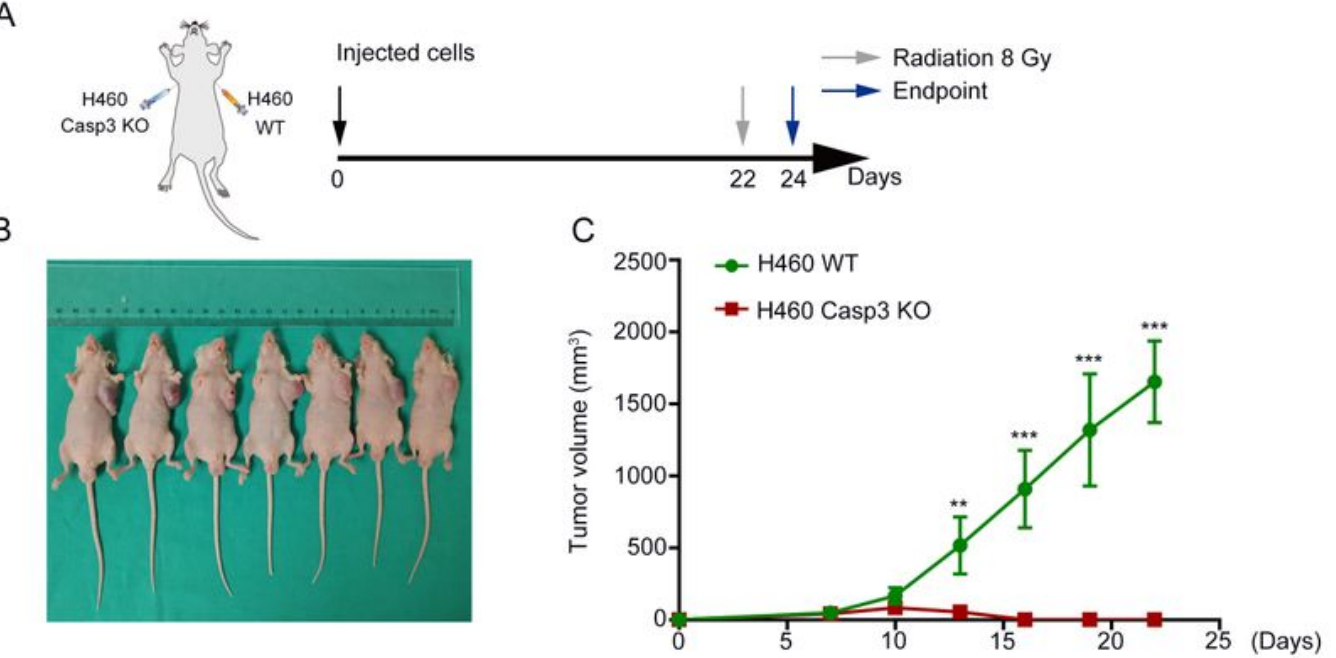

D
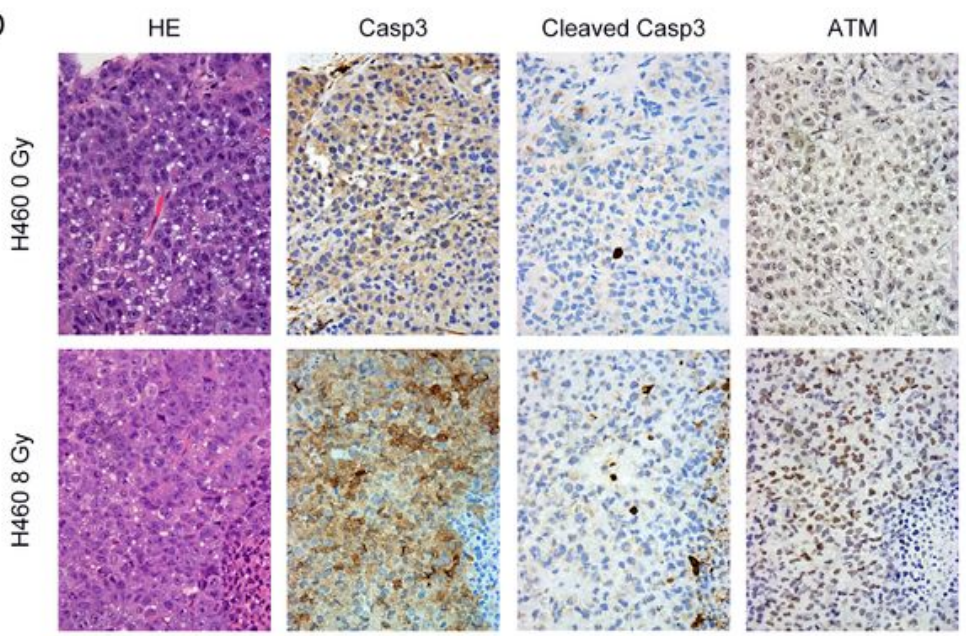

pATM (S1981)
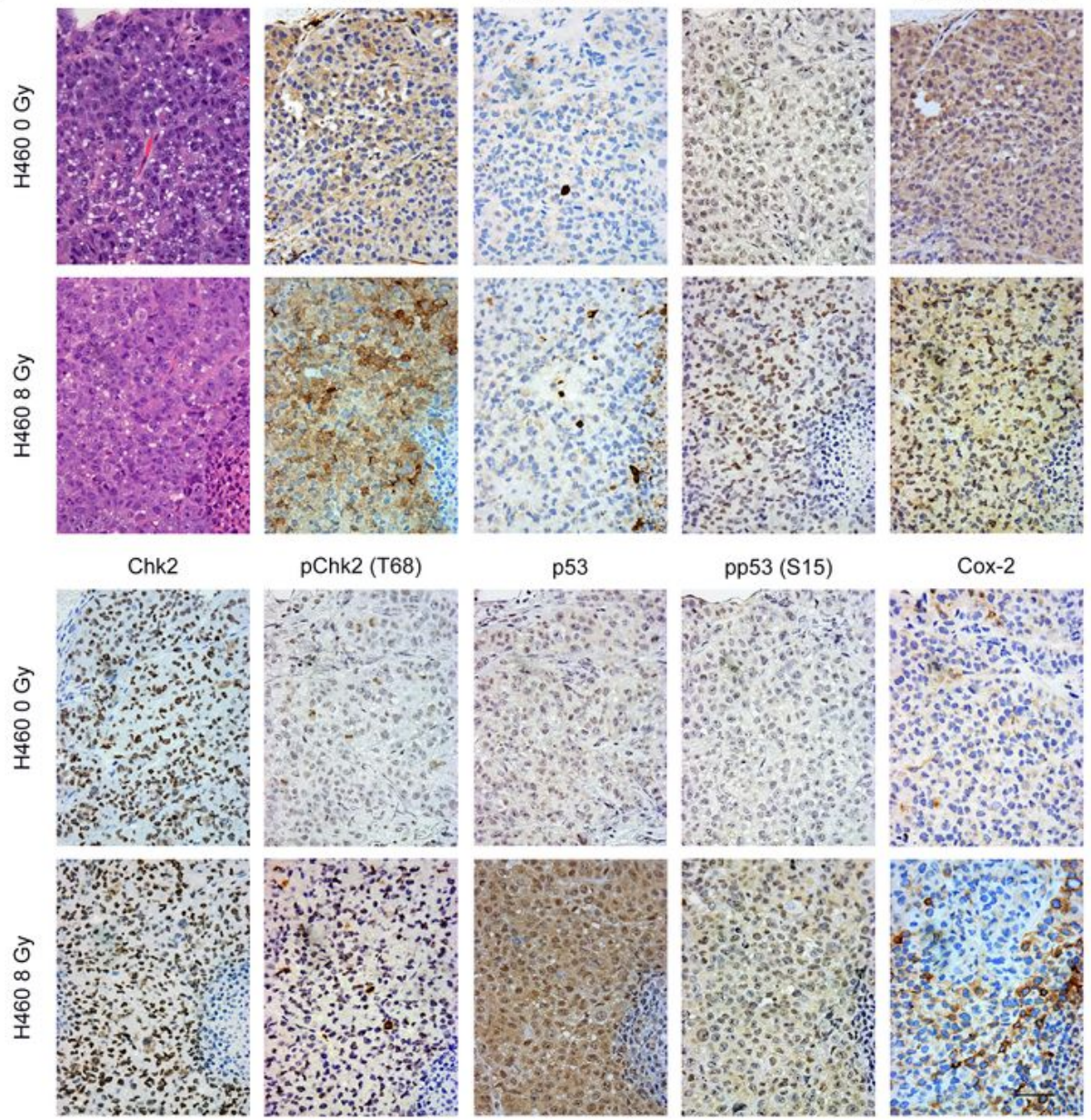

p53

$$
\text { pp53 (S15) }
$$
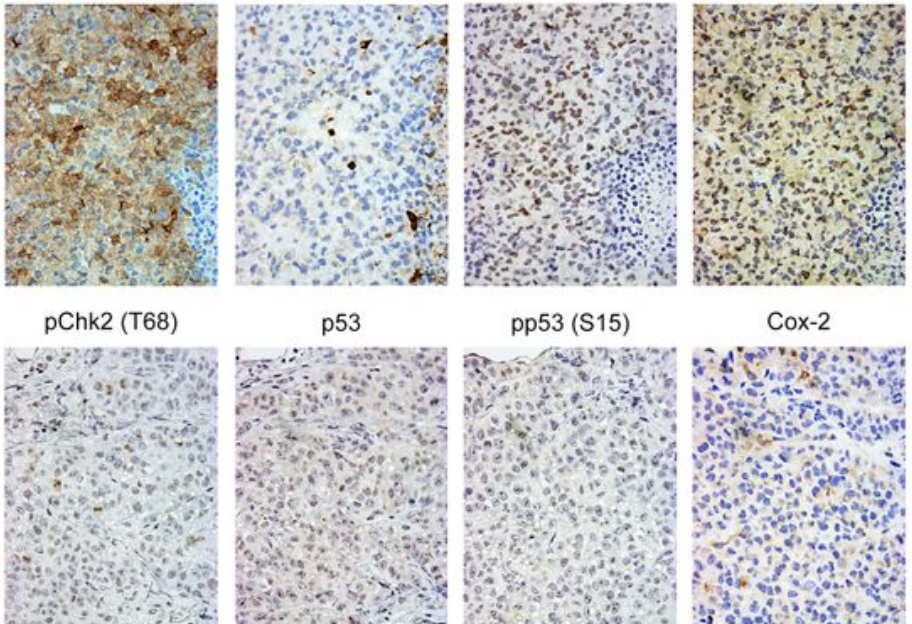

Cox-2
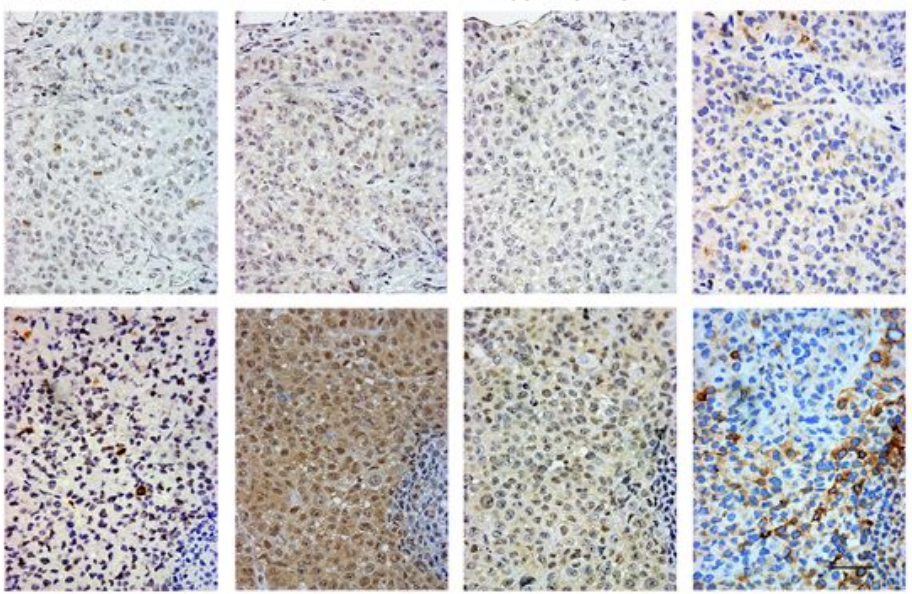

\section{Figure 6}

Casp3 KO restrains tumor formation and radiation activates ATM/p53/Cox-2 axis in vivo. A. Treatment scheme for nude mice. B. Images of tumor size were obtained on day 22. C. The tumor volume of xenografts was measured with calipers every 2 or 3 days ( ${ }^{*} p<0.01,{ }^{\star *} p<0.001$, Student's $t$ test, $\left.n=7\right)$. D. Representative photomicrographs of H\&E and immunohistochemical staining of caspase-3, Cleaved 
caspase-3, ATM, pATM (S1981), Chk2, pChk2 (T68), p53, pp53 (S15) and Cox-2 in tumor tissues. Scale bars: $50 \mu \mathrm{m}$.

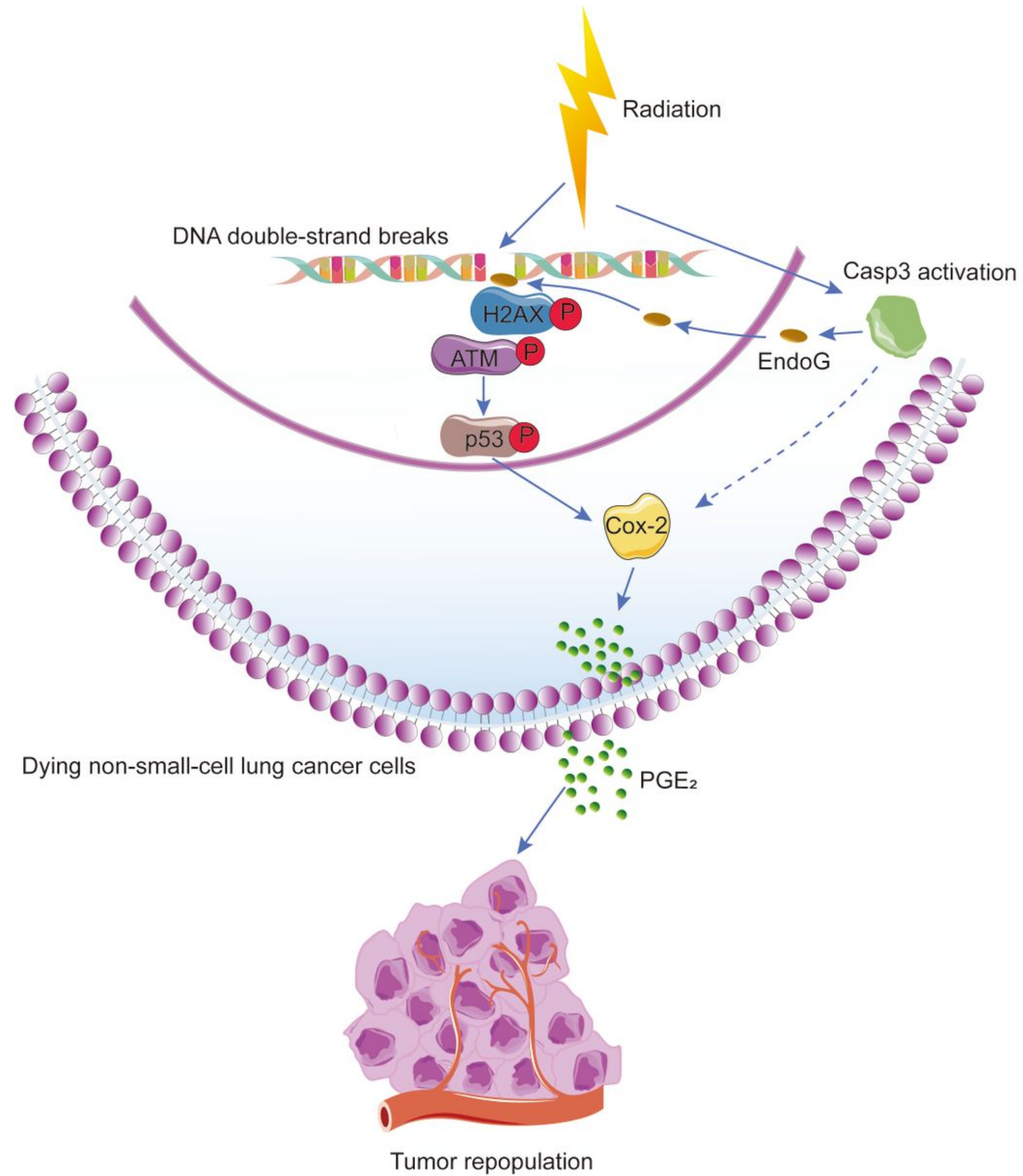

\section{Figure 7}

Diagram illustrating the proposed mechanism of radiation-induced tumor repopulation in NSCLC. Radiation-induced DNA DSBs activates the DNA damage response (DDR) and caspase-3. Activated caspase-3 regulates EndoG nuclear translocation, thus participates in the DDR via regulating ATM/p53 
signaling, which brings to the activation of Cox-2/PGE2 axis in dying NSCLC cells, consequently enhances living tumor cells proliferation.

\section{Supplementary Files}

This is a list of supplementary files associated with this preprint. Click to download.

- Additionalfile1.doc

- Fig.S1.tif

- Fig.S2.tif

- Fig.S1.tif

- Fig.S2.tif

- Fig.S3.tif

- Fig.S4.tif

- Additionalfile1.doc

- Fig.S3.tif

- Fig.S4.tif 